\title{
An Optimized Herbal Formula Reverses the Hepatotoxicity Induced by Acetaminophen
}

\author{
Manjir Sarma Kataki*, Bibhuti Bhusan Kakoti \\ Department of Pharmaceutical Sciences, Dibrugarh University, Dibrugarh, Assam, INDIA.
}

\begin{abstract}
Objectives: In this present study the hepatoprotective effect of an optimized herbal formula (HF) was appraised against paracetamol induced liver damage in rats. Methods: In vitro antioxidant activity of the HF was evaluated by ABTS radical scavenging assay. The hepatoprotective activity of HF $(100,200$ and $400 \mathrm{mg} / \mathrm{kg} \mathrm{b.w)}$ was assessed against paracetamol induced liver damage in rats. Serum enzymatic levels of serum glutamate oxaloacetate transaminase (AST), serum glutamate pyruvate transaminase $(A L T)$, serum alkaline phosphatase (ALP) and Gamma-glutamyltransferase $(\gamma$-GT) were appraised along with estimation of catalase (CAT), superoxide dismutase (SOD), Glutathione (GSH) and TBARS levels in liver tissues. Lipid profile was also examined. Furthermore, histopathological examination of the liver sections was performed to approve and advocate the induction of hepatotoxicity as well as hepatoprotective effectiveness. Results: The HF showed robust in vitro antioxidant activities in terms of ABTS radical scavenging. HF reinstated the significantly raised serum enzymatic levels of AST, ALT, ALP and $\gamma$-GT in a dose dependent manner. The lipid profile
\end{abstract}

was also found to be stabilized. The histopathological remarks did further establish the biochemical indications of hepatoprotection. Elevated level of catalase (CAT), superoxide dismutase (SOD), Glutathione (GSH) and reducedTBARS levels in liver tissues further reinforce the hepatoprotective actions. Conclusion: The outcomes evidently unveil the antioxidant efficacy and hepatoprotective activity of HF against paracetamol induced liver damage in rats.

Key words: Antioxidant, Hepatoprotective, Herbal formula, Paracetamol, Silymarin.

Correspondence

Dr. Manjir Sarma Kataki,

Research Scholar, Department of Pharmaceutical Sciences, Dibrugarh University, Dibrugarh-786004, Assam, INDIA.

Email id: manjirkataki@gmail.com

DOI: $10.5530 /$ jyp.2022.14.11

\section{INTRODUCTION}

The liver is the main regulatory organ responsible for metabolism of all the xenobiotics as well as synthesis and productions of numerous chemical and enzymes. Hepatic parenchymal injuries are at all times demonstrated to be related to the disruption of diverse liver metabolic pathways and functions. ${ }^{1}$ There are plentiful aetiologic modalities as well as numerous infectious mediators, several chemical agents, viruses, and environmental toxins accountable for triggering hepatotoxicity and liver impairment. The growing published literatures also suggested the significant role of free radicals and oxidative stress in the pathophysiology of numerous liver ailments. Many experimental hepatotoxins like carbon tetrachloride, paracetamol etc. were found to be linked with the generation of free radicals and oxidative stress which further were proved to be the mechanisms by which these toxins caused hepatic damage. ${ }^{1,2}$ These mechanisms became the pathophysiological basis of numerous liver diseases together with hepatic fibrosis, hepatic injury, necrosis and apoptosis. ${ }^{2}$

Furthermore, superoxide dismutase (SOD), catalase (CAT) and glutathione peroxidase (GSH) etc. as part of the physiological antioxidant defense system, used to maintain a protective status in the physiology via neutralizing free radicals time to time. Therefore, any disturbance in this enzyme systems predisposed the physiological system to damage. In addition, vitamin scarcity together with swarming of free radicals and a diminished level of above-mentioned enzymes, is highlighted as the leading offender for producing oxidative stress. ${ }^{3,4}$ Considering these concepts, the current growing research was focused upon the neutralization of free radicals and the prevention of lipid peroxidation as well as restoration of the antioxidant enzyme system and these modalities have been beleaguered as the likely treatment and prevention goal of hepatotoxicity and other liver ailments. ${ }^{5,6}$

Another concerns or gap as evident from literatures indicated a scarcity of hepatoprotective treatment or management modality and also suggested the very adverse reactions of the available hepatoprotectives.? Therefore, the search for a new hepatoprotective form natural origin without any significant side effects but with better safety profile, has been the new-fangled research activities in the field. There is a rising emphasis upon natural antioxidants against chemically or drug induced toxicities. In view of the necessity and mounting concerns, an herbal formula has been developed and optimized in our pharmacological laboratory which comprised of mixture of methanol extracts of four different indigenous plants namely of Rumex hastetus, Alternanthera sessilis, Angelica sinensis and Urtica dioica. This present study was intended to study the pharmacologic activity of the optimized herbal formula (HF) in terms of antioxidant as well hepatoprotective efficacy in paracetamol induced rat model of hepatotoxicity.

\section{MATERIALS AND METHODS}

\section{Chemicals and Drugs}

Silymarin was received as gift sample from Ranbaxy Laboratories, Delhi, India. Malondialdehyde (MDA) and paracetamol were procured from Sigma Chemicals Company, St Louis, MO, USA and E Merck, Mumbai, India, respectively. All other chemical and reagents arranged, were of analytical grade and purchased from reputed vendors. 


\section{Acute toxicity studies}

An acute oral toxicity study was accomplished using three Wistar rats of either sex as per the OECD (Organization of Economic Co-operation and Development) guidelines for the testing of chemicals, Test No. 423 (OECD, 2001; Acute oral toxicity-Acute toxic class method). The rats were fasted overnight prior to the experimentation. The HF was orally ingested in cumulative dose up to $2000 \mathrm{mg} / \mathrm{kg}$.

\section{In vitro antioxidant activity}

The free radical scavenging activity of the HF was appraised by using ABTS radical scavenging model described previously. ${ }^{8}$

\section{In vivo hepatoprotective activity}

The in vivo hepatoprotective activity was evaluated in acetaminophen induced hepatotoxicity model using Wister rats.

\section{Animals}

Wistar rats (180-240 g) of either sex were acquired from the S/N Chakraborty Enterprise, Kolkata, West Bengal, India (Reg. No. 1576/ GO/a/11/CPCSEA) for the study. The animals were kept in big, hygienic polypropylene cages in a temperature-controlled room $\left(22 \pm 2^{\circ} \mathrm{C}\right.$ with relative humidity (45-55\%) under $12 \mathrm{hr}$ light and dark cycles. All the animals were accustomed to laboratory conditions for two weeks prior to experimentation. Animals were provided with a standard pellet diet and fresh drinking water ad libitum. The care and use of laboratory animals were strictly according to the guidelines prescribed by the Institutional Animal Ethical Committee (Approval no. IAEC/DU/63) constituted under the guidelines of Committee for the Purpose of Control and Supervision of Experiments on Animals (CPCSEA), India.

\section{Experimental design}

A total of 34 rats were divided into six groups where group II and III contained 5 rats each and other groups contained 6 rats each. ${ }^{9}$

- Group I served as normal control and received the vehicle (50\% propylene glycol; p.o.) and Olive oil ( $2 \mathrm{ml} / \mathrm{kg}$, p.o.) for 15 days

- Group II received Olive oil (2 ml/kg, p.o.) for 15 days and paracetamol (640 mg/kg, p.o.) $24 \mathrm{hr}$ after the last dose.

- Group III received silymarin $100 \mathrm{mg} / \mathrm{kg}$ orally (p.o.) for 15 days and paracetamol (640 mg/kg, p.o.) $24 \mathrm{hr}$ after the last dose.

- Groups IV, V, VI were administered HF at 100, 200, and $400 \mathrm{mg} / \mathrm{kg}$ body weight p.o. respectively for 15 days and paracetamol $(640 \mathrm{mg} / \mathrm{kg}$, p.o.) $24 \mathrm{hr}$ after the last dose.

\section{Body weight gain}

The body weight gain was logged by deducting the final bodyweight from the initial body weight. After 5 days of treatments, animals which were anaesthetized and fasted overnight were decapitated and blood samples were withdrawn in sterilized bare tubes.

\section{Blood and tissue sampling}

Serum was stored at $-20^{\circ} \mathrm{C}$ for estimation of lipid profile and for performing further biochemical estimations. Liver was removed, washed with ice cold phosphate buffer saline, dehydrated using filter paper and weighed. Liver for each rat was divided into 5 fragments; 3 of which were kept at $-80^{\circ} \mathrm{C}$ until preparation of liver homogenate for oxidative stress marker CAT, GSH, SOD and TBARS. The remaining portions were kept engrossed in $10 \%$ neutral buffered formalin for histopathological examinations.

\section{Lipid profile and Liver Functions}

Cholesterol, total cholesterol (TC), Serum triglycerides (TG), low-density lipoprotein (LDL) and high-density lipoprotein (HDL) were estimated using enzymatic calorimetric kits. ${ }^{10}$ The serum alanine transaminase (ALT), serum aspartate transaminase (AST), alkaline phosphatase (ALP) and Gamma-glutamyltransferase $(\gamma-\mathrm{GT})$ were estimated employing the Hitachi 912 clinical chemistry automatic analyzer (Roche Diagnostic $\mathrm{GmbH}$, Mannheim, Germany).

\section{Estimation of oxidative stress markers}

The biochemical markers indicating oxidative stress were measured in homogenised liver tissue. Superoxide dismutase (SOD) was also estimated by the technique designated earlier. ${ }^{11,12}$ Catalase (CAT) content in liver homogenate was measured by the method described previously. ${ }^{13}$ GSH activity in liver homogenate was estimated at absorbance of 412 $\mathrm{nm}$ employing calorimetric kit (BioVision, USA) as per the method described previously. ${ }^{14}$

\section{Appraisal of thiobarbituric acid reactive substances (TBARS)}

Thiobarbituric acid reactive substances (TBARS) contents were estimated by a method described previously. ${ }^{15}$

\section{Histopathology}

Histopathological evaluations were performed further. Formalin fixed liver sections were arranged using standard measures for Hematoxylin and Eosin stain as designated elsewhere. ${ }^{16,17}$

\section{Statistical analysis}

The data were articulated as mean \pm SD. Statistical variances at $p<0.05$ between the groups were scrutinized by one-way ANOVA followed by Turkey as post hoc using GraphPad Instat software bundle. The $\mathrm{IC}_{50}$ value was calculated graphically by linear regression analysis. All the analyses were done using GraphPad Prism (Version 5.01, GraphPad Software, San Diego, USA).

\section{RESULTS}

\section{Acute toxicity studies}

The acute toxicity study demonstrated safety of the HF upto a maximum dose of $2000 \mathrm{mg} / \mathrm{kg}$.

The HF did not produce any mortality upto $2000 \mathrm{mg} / \mathrm{kg}$ dose level. Henceforward $1 / 20^{\text {th }}, 1 / 10^{\text {th }}$ and $1 / 5^{\text {th }}$ of the maximum dose (i.e., 100, 200 and $400 \mathrm{mg} / \mathrm{kg}$, p.o.) were carefully chosen for the present study. No mortality as well as any significant toxic reactions were observed by $2000 \mathrm{mg} / \mathrm{kg}$ dose level.

\section{In vitro antioxidant activity}

Figure 1 shows the decline in concentration of ABTS radical owing to scavenging capability of HF and standard compound (BHT) at various concentration levels $(10-250 \mu \mathrm{g} / \mathrm{ml})$. A similar as well as equivalent scavenging capacity of $\mathrm{HF}$ and $\mathrm{BHT}$ on ABTS radical was noted. The percent ABTS scavenging activity of $\mathrm{HF}$ and BHT were noted to be 97.47 and $99.37 \%$, respectively at the concentration of $250 \mu \mathrm{g} / \mathrm{ml}$. The outcomes presented $\mathrm{HF}$ as a robust scavenger of ABTS radical analogous to standard BHT. The $\mathrm{IC}_{50}$ values were also calculated for $\mathrm{HF}$ and $\mathrm{BHA}$ employing linear regression analysis. The $\mathrm{IC}_{50}$ values of $\mathrm{HF}$ and $\mathrm{BHT}$ were noted to be $94.30 \mu \mathrm{g} / \mathrm{ml}$ and $78.21 \mu \mathrm{g} / \mathrm{ml}$, respectively.

\section{In vivo hepatoprotective activity}

\section{Body weight gain and Liver weight}

Paracetamol produced significant diminution $(p<0.0001)$ in body weight whereas augmented the absolute liver weight $(p<0.0001)$ comparatively to control group. The augmented absolute liver weight by paracetamol intoxication was found to be suggestively $(p<0.0001)$ reduced and 


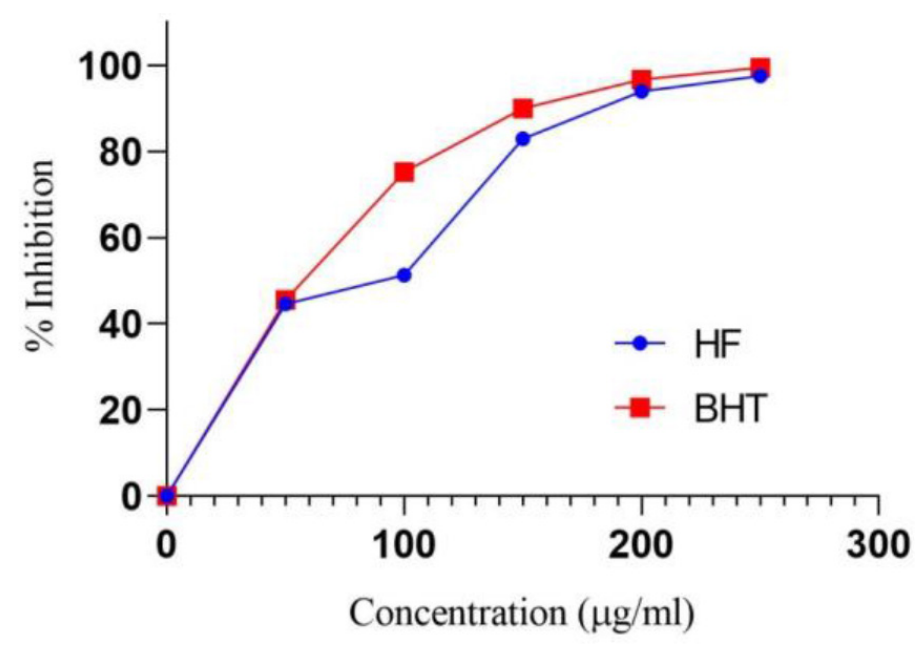

Figure 1: ABTS radical scavenging activity in $\mathrm{HF}(n=3)$.

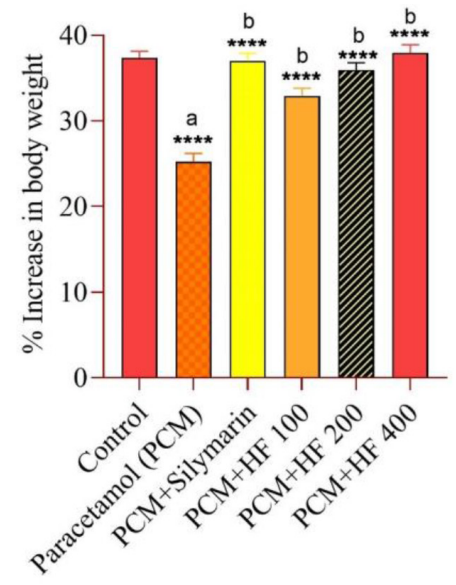

Treatment Groups

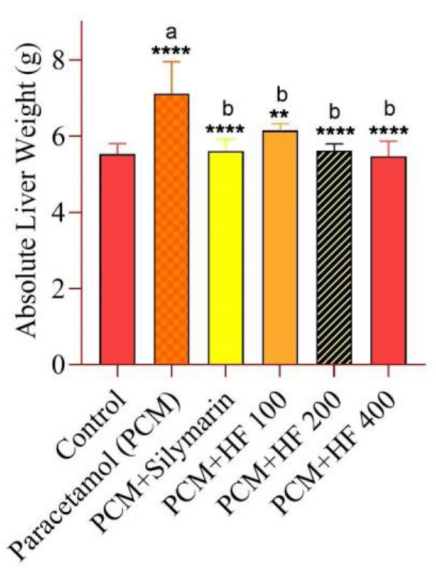

Treatment Groups
Figure 2: Body weight gain and absolute liver weight in percentage. a indicate significance from the control group at $p<0.0001$ probability level. $b$ indicate significance from the Paracetamol (PCM) group at ${ }^{* *} p<0.01$, ${ }^{* * *} P<0.0001$ probability level.

reinstated with HF treatment (Figure 2). HF demonstrated a dose dependent restoration in the body weight $(p<0.0001)$.

\section{Lipid profile and Liver Functions}

Paracetamol treatment caused a significant rise in serum triglyceride (TG), serum total cholesterol (TC), and low-density lipoprotein (LDL) levels $(p<0.0001)$, whereas high density lipoprotein (HDL) level was found to be reduced significantly $(p<0.001)$ compared to control group animals. Treatment with $\mathrm{HF}$ at 200 and $400 \mathrm{mg} / \mathrm{kg}$ dose levels significantly reduced and restored the serum triglyceride (TG), serum total cholesterol (TC), and low-density lipoprotein (LDL) levels $\left({ }^{* *} p<0.01\right.$ and $\left.{ }^{* * *} p<0.0001\right)$ as compared to paracetamol group animals. In addition, HDL level was found to be increased significantly by the HF treatment as compared to paracetamol group. However, HF at $100 \mathrm{mg} / \mathrm{kg}$ dose level did not cause any significant rise in HDL level (Not significant). The results are presented in Table 1.
The hepatoprotective effects of HF in terms of liver function parameters are presented in Table 2. The liver profile tests including AST, ALT, ALP and $\gamma$-GT levels were observed to be increased by the treatment of paracetamol as compared to normal control animals, however, HF administration at all the studied dose levels (100, 200 and $400 \mathrm{mg} / \mathrm{kg}$ ) demonstrated significant

decrease and restoration of the AST, ALT, ALP and $\boldsymbol{\gamma}$-GT levels to normal as compared to paracetamol group animals $(p<0.0001)$.

\section{Assessment of oxidative stress markers}

Paracetamol treatment in rats significantly reduced the activity of CAT, SOD, GSH $(p<0.01)$ whereas amplified TBARS levels $\left({ }^{* * *} p<0.0001\right)$ in liver tissues as compared to normal control rats. Generation of free radical and oxidative stress increased the lipid peroxidation as evident by higher levels of TBARS, leading to diminution in the antioxidant defense system in terms of reduction in the levels of CAT, SOD and GSH. The $\mathrm{HF}$ administration significantly increased and restored the antioxidant defense enzyme system by restoring the CAT, SOD and GSH levels to normal. The HF treatment at all dose levels $(100,200$ and $400 \mathrm{mg} / \mathrm{kg} \mathrm{bw})$ showed significant upsurge of CAT, SOD and GSH levels as compared to

Table 1: Effect of HF on Lipids profile.

\begin{tabular}{lcccc}
\hline & TG $(\mathrm{mg} / \mathrm{dl})$ & $\mathrm{TC}(\mathrm{mg} / \mathrm{dl})$ & $\mathrm{HDL}(\mathrm{mg} / \mathrm{dl})$ & $\mathrm{LDL}(\mathrm{mg} / \mathrm{dl})$ \\
\hline Control & $6.83 \pm 0.49$ & $5.14 \pm 0.29$ & $4.79 \pm 0.27$ & $3.67 \pm 0.30$ \\
Paracetamol & $10.14 \pm 0.57 \# \# \# \#$ & $10.23 \pm 0.31 \# \# \#$ & $3.31 \pm 0.22 \# \# \#$ & $9.62 \pm 0.16 \# \# \# \#$ \\
$\begin{array}{l}\text { Silymarin + } \\
\text { Paracetamol }\end{array}$ & $7.09 \pm 0.42^{* * * *}$ & $5.42 \pm 0.41^{* * * *}$ & $4.71 \pm 0.61^{* *}$ & $4.04 \pm 0.07^{* * * *}$ \\
HF 100 mg + & $9.10 \pm 0.56^{*}$ & $8.09 \pm 0.51^{* * * *}$ & $4.19 \pm 0.55 \mathrm{~ns}$ & $8.00 \pm 0.06^{* * * *}$ \\
Paracetamol & & & & \\
HF 200 mg + & $7.11 \pm 0.47^{* * * *}$ & $6.14 \pm 0.43^{* * *}$ & $4.53 \pm 0.75^{* *}$ & $6.05 \pm 0.09^{* * * *}$ \\
Paracetamol & & & & \\
HF 400 mg + & $6.84 \pm 0.81^{* * * *}$ & $5.27 \pm 0.37^{* * * *}$ & $4.75 \pm 0.72^{* *}$ & $4.10 \pm 0.09^{* * * *}$ \\
Paracetamol & & & & \\
\hline
\end{tabular}

Mean $\pm \mathrm{SD}$ ( $\mathrm{n}=6$ number).

$\# \# \mathrm{p}<0.001, \# \# \# \mathrm{p}<0.0001$ probability level indicate significance from the control group.

${ }^{*} \mathrm{p}<0.05,{ }^{* *} \mathrm{p}<0.01,{ }^{* *} \mathrm{p}<0.001,{ }^{* * *} \mathrm{P}<0.0001$ probability level indicate significance from the paracetamol group.

Table 2: Effect of HF on hallmarks of hepatotoxicity.

\begin{tabular}{ccccc}
\hline & $\begin{array}{c}\text { ALT (SGPT) } \\
\text { (IU/L) }\end{array}$ & $\begin{array}{c}\text { AST (SGOT) } \\
\text { (IU/L) }\end{array}$ & $\begin{array}{c}\text { ALP } \\
\text { (IU/L) }\end{array}$ & $\begin{array}{c}\text { Y-GT (nM/min / } \\
\text { mg protein) }\end{array}$ \\
\hline Control & $60.18 \pm 2.47$ & $81.31 \pm 1.33$ & $183.22 \pm 3.66$ & $110.24 \pm 2.32$
\end{tabular}

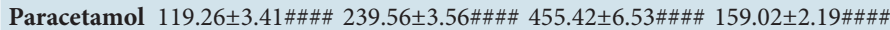
Silymarin $+64.50 \pm 1.25^{* * * *} \quad 90.85 \pm 1.52^{* * * *} \quad 191.63 \pm 2.62^{* * *} \quad 114.97 \pm 3.22^{* * * *}$ Paracetamol

HF $100 \mathrm{mg}+93.82 \pm 1.29^{* * * *} \quad 152.76 \pm 2.78^{* * * *} \quad 306.67 \pm 2.75^{* * * *} \quad 132.87 \pm 3.18^{* * * *}$ Paracetamol

HF $200 \mathrm{mg}+71.80 \pm 1.38^{* * * *} \quad 95.54 \pm 2.20^{* * * *} \quad 210.10 \pm 2.82^{* * * *} \quad 116.09 \pm 3.32^{* * * *}$ Paracetamol

HF $400 \mathrm{mg}+63.65 \pm 1.71^{* * * *} \quad 87.65 \pm 1.07^{* * * *} \quad 194.65 \pm 2.86^{* * * *} 113.22 \pm 3.12^{* * * *}$ Paracetamol

Mean $\pm \mathrm{SD}$ ( $\mathrm{n}=6$ number).

$\# \# \#$ p $<0.0001$ probability level indicate significance from the control group.

${ }^{* * *} \mathrm{p}<0.0001$ probability level indicate significance from the $\mathrm{CCl} 4$ group. 
Table 3: Effect of HF on markers of oxidative stress.

\begin{tabular}{ccccc}
\hline & $\begin{array}{c}\text { CAT } \\
(\mathrm{U} / \mathrm{min})\end{array}$ & $\begin{array}{c}\text { SOD } \\
(\mathrm{U} / \mathrm{mg} \text { protein) }\end{array}$ & $\begin{array}{c}\text { GSH } \\
(\mu \mathrm{g} \text { tissue })\end{array}$ & $\begin{array}{c}\text { TBARS } \\
(\mathrm{nM} / \mathrm{min} / \mathrm{mg} \\
\mathrm{protein})\end{array}$ \\
\hline Control & $4.09 \pm 0.86$ & $22.62 \pm 1.02$ & $3.86 \pm 0.02$ & $29.21 \pm 1.11$ \\
Paracetamol & $2.64 \pm 0.12 \# \#$ & $11.82 \pm 0.82 \# \# \#$ & $0.43 \pm 0.01 \# \# \# \# 3.07 \pm 1.32 \# \# \# \#$ \\
$\begin{array}{c}\text { Silymarin + } \\
\text { Paracetamol }\end{array}$ & $4.61 \pm 1.12^{* * *}$ & $20.79 \pm 0.85^{* * * *}$ & $3.75 \pm 0.58^{* * * *}$ & $27.97 \pm 1.41^{* * * *}$ \\
HF 100 mg + & $4.36 \pm 0.94^{* *}$ & $16.43 \pm 0.96^{* * * *}$ & $2.94 \pm 0.48^{* * * *}$ & $40.94 \pm 1.23^{* * * *}$ \\
Paracetamol & & & & \\
HF 200 mg + & $4.56 \pm 0.87^{* * *}$ & $19.52 \pm 0.97^{* * * *}$ & $3.67 \pm 0.54^{* * * *}$ & $32.09 \pm 1.16^{* * * *}$ \\
Paracetamol & & & & \\
HF 400 + PCM & $4.65 \pm 0.92^{* * *}$ & $1.94 \pm 0.92^{* * * *}$ & $3.81 \pm 0.41^{* * * *}$ & $28.18 \pm 1.13^{* * * *}$ \\
\hline
\end{tabular}

Mean $\pm \mathrm{SD}$ ( $\mathrm{n}=6$ number).

$\# \# \mathrm{p}<0.01, \# \# \# \mathrm{p}<0.0001$ probability level indicate significance from the control group.

${ }^{* *} \mathrm{p}<0.01,{ }^{* * *} \mathrm{p}<0.001,{ }^{* * *} \mathrm{p}<0.0001$ probability level indicate significance from the paracetamol group.

paracetamol group animals $\left({ }^{* *} p<0.01,{ }^{* *} p<0.001,{ }^{* * *} p<0.0001\right)$. The results are presented in Table 3.

\section{Liver histopathology}

The shielding and hepatoprotective outcome demonstrated by HF against paracetamol induced hepatotoxicity was further confirmed and strengthen by histopathological evaluation. Histopathological observations displayed that the normal architecture of liver was significantly lost paracetamol treated rats with the presence of fatty changes, vacuolization, and necrosis of hepatocytes in the central lobular area (Figure 3). An extensive liver injury marked by moderate to severe fibrosis, hepatocellular hydropic degeneration, necrosis and mononuclear inflammatory leukocyte infiltrations have been evident in the paracetamol treated rats. Nevertheless, treatment by Silymarin and HF markedly amended the hepatic lacerations produced by paracetamol. The fibrotic tissues were seen as blue colour by Masson's trichrome stain. In normal control animals, the liver sections showed normal hepatic cells without any sign of fibrosis or necrosis (a). The livers of paracetamol treated rats showed prevalent fibrotic tissue compared to the normal control (b). Furthermore, HF treatment at $400 \mathrm{mg} / \mathrm{kg}$ dose level (d) confirmed significant protection from the signs of paracetamol intoxication and produced more or less normal lobular form and a minor fatty change, and necrosis almost comparable to the control and silymarin treated groups (c).

\section{DISCUSSION}

It has long been recognized that paracetamol causes liver toxicity if taken in excess of therapeutic levels. Paracetamol remains a major cause of acute hepatotoxicity in the world due to its increasing prevalence in combination medications for pain relief and antihistamines. When paracetamol is co-ingested with other medications, the rise in serum paracetamol levels may be delayed, which can alter medical management. An update is provided here on the current available knowledge and treatment options concerning the hepatotoxicity caused by paracetamol. $\mathrm{N}$ acetyl-p-benzoquinone imine was believed to be responsible for acetaminophen's hepatotoxicity; however, the mechanism by which the metabolite caused it was unknown. Having an electrophile nature and being a potent oxidizing agent, the metabolite could covalently bind to various important proteins, resulting in oxidative stress. ${ }^{18}$ Literatures
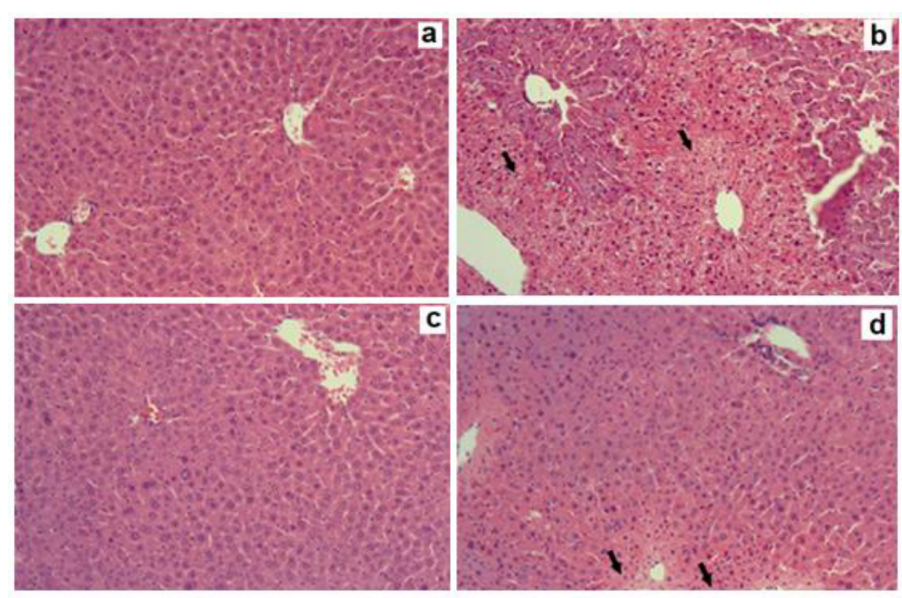

Figure 3: Histopathological photomicrographs of liver sections. a) Normal control, b) Paracetamol control, c) Silymarin treated, d) HF 400 mg/kg bw treated.

suggested that in paracetamol intoxication covalent binding correlates with the toxicity; however, as has been pointed out many other studies covalent binding has been simply an assay for the reactive metabolite of paracetamol and the reactive metabolite might cause the toxicity by other mechanisms not known yet. However, the most cited and well-known theory of paracetamol hepatotoxicity indicated the role of oxidative stress as the main culprit. The reactive metabolite ( $\mathrm{N}$ acetyl-p-benzoquinone imine) was an oxidizing agent that exhausted the cell of glutathione, a cellular protectant against oxidative stress. Oxidative stress was normally linked with production of a reactive oxygen species: hydrogen peroxide, superoxide anion, and the highly reactive hydroxyl radical. These species were formed by consecutive one-electron reduction of molecular oxygen. This mechanism might lead to lipid peroxidation, protein oxidation, GSH oxidation and DNA damage and iron may be significant in their catalytic formation. Though, it is not clear how reactive oxygen may be produced in vivo by a metabolite of acetaminophen. Considering all these associations, these markers were well studied and evaluated in the present study. The present study revealed the hepatoprotective and antioxidant effects of HF against paracetamol induced liver injury in rats. There are several biomarkers of lipid peroxidation like peroxide products including Malonaldehyde (MDA), TBARS etc., which can be estimated as a hallmark sign of cellular damage or hepatic injury. ${ }^{19-21}$ A surfeit of research revealed oxidative stress as an important pathophysiological modality indicative of cellular injury leading to numerous disease conditions. ${ }^{22-24}$ In this context, antioxidant potential is observed as one of the significant parameters suggestive of the likely mechanism of hepatoprotection.

The HF significantly demonstrated the ABTS scavenging activity suggesting free radical neutralizing potential of the herbal formula. The $\mathrm{IC}_{50}$ values of HF and BHT were found to be $94.30 \mu \mathrm{g} / \mathrm{ml}$ and $78.21 \mu \mathrm{g} / \mathrm{ml}$, respectively, indicating the significant free radical neutralizing ability of HF. In necrosis or hepatic damage, serum hepatobiliary enzymes (AST, ALT, ALP and $\gamma$-GT) leak into the blood circulation; indicating a significant increase in serum levels of these enzymes. ${ }^{25}$ In paracetamol treated animals, the cellular injury caused an elevation in the serum AST, ALT, ALP and $\boldsymbol{\gamma}$-GT levels, indicative of injury and disruption of functional integrity of cell membranes in liver. ${ }^{26,27}$ Elevated TBARS confirmed the proclaimed hepatic damage. Moreover, physiological antioxidant defense enzyme system and their protective mechanism 
was found to be failed as evident from the diminished estimation of CAT, SOD and GSH in paracetamol treated animals. There were noted restoration of the physiological antioxidant defense enzyme system by $\mathrm{HF}$ as indicated by the rise in the CAT, SOD and GSH levels. The decrease in the levels of TBARS in the HF treated animals also indicated the significant hepatoprotection. These results clearly revealed the significant hepatoprotective activity of HF as suggested by the various biochemical markers and estimations. Treatment with HF in different animal groups revealed a significant decrease and restoration in serum AST, ALT, ALP and $\gamma$-GT levels as compared to paracetamol treated group. The extent of hepatic necrosis and the parallel leakage of hepatic intracellular enzymes were found to be inhibited by stabilizing hepatic cellular membranes by the treatment of HF.

Another sign of hepatotoxicity was the abnormality in the lipid profile as indicated by the elevated measurement of serum levels of cholesterol (TC), triglyceride (TG) and low-density lipoprotein (LDL) as well as significant decrease in serum HDL levels in the paracetamol treated animals. The HF significantly normalized the lipid profile by reducing the serum levels of cholesterol (TC), triglyceride (TG) and low-density lipoprotein (LDL) as well as significant increased the serum HDL levels. There was also observed a significant inhibition of degree of lipid peroxidation by HF as advised by the results of the thiocyanate method in the total antioxidant activity assay.

Furthermore, histopathological findings and observations confirmed the hepatoprotective activity of the HF. Paracetamol demonstrated prevalent liver injuries characterized by moderate to severe necrosis, fibrosis, hepatocellular hydropic degeneration and signs of leukocyte infiltrations. $\mathrm{HF}$ at $400 \mathrm{mg} / \mathrm{kg}$ demonstrated significant hepatoprotection as evident by restoration of the liver architecture as well as reversal of the severe hepatic fibrosis induced by paracetamol. The results were in agreement with the results of the oxidative damage investigation. Ultimately, the results of this present study indicated the herbal formula as a significant hepatoprotective and a significant antioxidant with credible as well as reliable mechanisms.

\section{CONCLUSION}

The present study undeniably exhibited the 'in vitro' antioxidant activity and 'in vivo' hepatoprotective efficacy of the herbal formula. The herbal formula at all doses $(100,200$ and $400 \mathrm{mg} / \mathrm{kg})$ established significant hepatoprotective activity well evinced by the several biochemical markers as well as histopathological comments.

\section{ACKNOWLEDGEMENT}

The authors are grateful to the Department of Pharmaceutical Sciences, Dibrugarh University for all the possible support and help during the work. The authors are also thankful to Dr. D. N. Sharma, Ex. Director, Research Institute in ISM, Himachal Pradesh, for his valuable advice and timely support.

\section{CONFLICT OF INTEREST}

The authors declare that there is no conflict of interest.

\section{ABBREVIATIONS}

HF: Herbal Formula; SOD: Superoxide Dismutase; CAT: Catalase; GSH: Glutathione Peroxidase; MDA: Malondialdehyde; OECD: Organisation for Economic Co-operation and Development; ABTS: 2,2'-azino-bis (3-ethylbenzothiazoline-6-sulfonic acid; CPCSEA: Committee for the Purpose of Control and Supervision of Experiments on Animals; IAEC: Institutional Animal Ethic Committee; TBARS: Thiobarbituric Acid Reactive Substances; TC: Total Cholesterol; TG: Serum Triglycerides;
LDL: Low-Density Lipoprotein; HDL: High-Density Lipoprotein; ALT: Serum Alanine Transaminase; AST: Serum Aspartate Transaminase; ALP: Alkaline Phosphatase; $\gamma$-GT: Gamma-Glutamyltransferase; ANOVA: Analysis of Variance; BHT: Butylated Hydroxy Toluene; IC $_{50}$ : Half-maximal inhibitory concentration; PCM: Paracetamol; DNA: Deoxyribonucleic acid.

\section{REFERENCES}

1. Wolf PL. Biochemical diagnosis of liver diseases. Indian J Clin Biochem. 1999;14(1):59-90. doi: 10.1007/BF02869152, PMID 23105203.

2. Oh TY, Lee JS, Ahn BO, Cho H, Kim WB, Kim YB, et al. Oxidative damages are critical in pathogenesis of reflux esophagitis: Implication of antioxidants in its treatment. Free Radic Biol Med. 2001;30(8):905-15. doi: 10.1016/s08915849(01)00472-5, PMID 11295533.

3. Ellnain-Wojtaszek M, Kruczynski Z, Kasprzak J. Investigation of the free radical scavenging activity of Ginkgo biloba L. leaves. Fitoterapia. 2003;74(1-2):1-6. doi: 10.1016/s0367-326x(02)00306-4, PMID 12628386.

4. Halliwell B, Gutleridge M. Review article. Oxygen Toxic Oxygen Radic Transit Met Dis Bioche J. 1984;219:1-4

5. Gülçin I, Oktay M, Küfrevioglu OI, Aslan A. Determination of antioxidant activity of lichen Cetraria islandica (L.) Ach. J Ethnopharmacol. 2002;79(3):325-9. doi: 10.1016/s0378-8741(01)00396-8, PMID 11849836.

6. Masuda Y. [Learning toxicology from carbon tetrachloride-induced hepatotoxicity]. Yakugaku Zasshi. 2006;126(10):885-99. doi: 10.1248/ yakushi.126.885, PMID 17016019

7. Subramoniam A, Pushpangadan P. Development of phytomedicines for liver disease. Indian J Pharmacol. 1999;31:166-75.

8. Zheleva-Dimitrova D, Nedialkov P, Kitanov G. Radical scavenging and antioxidant activities of methanolic extracts from Hypericum species growing in Bulgaria. Pharmacogn Mag. 2010:6(22):74-8. doi: 10.4103/0973-1296.62889, PMID 20668569.

9. Devi KP, Sreepriya M, Balakrishna K, Devaki T. Protective effect of Premna tomentosa (L. Verbenaceae) extract on membrane-bound phosphatases and inorganic cations transport in acetaminophen-induced hepatotoxicity rats. J Ethnopharmacol. 2004;93(2-3):371-5. doi: 10.1016/j.jep.2004.04.010, PMID 15234779.

10. Finley PR, Tietz N. Clinical guide to laboratory tests. W B Saunders company; 1996.

11. Kakkar P, Das B, Viswanathan PN. A modified spectrophotometric assay of superoxide dismutase. Indian J Biochem Biophys. 1984;21(2):130-2. PMID 6490072.

12. Misra HP, Fridovich I. The Role of superoxide anion in the autoxidation of epinephrine and a simple assay for superoxide dismutase. J Biol Chem. 1972;247(10):3170-5. doi: 10.1016/S0021-9258(19)45228-9, PMID 4623845

13. Chance B, Maehly A. Assay of catalases and peroxidases; 1955.

14. Tietze F. Enzymic method for quantitative determination of nanogram amounts of total and oxidized glutathione: Applications to mammalian blood and other tissues. Anal Biochem. 1969;27(3):502-22. doi: 10.1016/0003-2697(69)90064-5, PMID 4388022

15. Iqbal M, Sharma SD, Rezazadeh $H$, Hasan N, Abdulla M, Athar M Glutathione metabolizing enzymes and oxidative stress in ferric nitrilotriacetate mediated hepatic injury. Redox Rep. 1996;2(6):385-91. doi: 10.1080/13510002.1996.11747079, PMID 27406673.

16. Hirayama C, Morotomi I, Hiroshige K. Quantitative and metabolic changes of hepatic collagens in rats after carbon tetrachloride poisoning. Biochem $\mathrm{J}$. 1970;118(2):229-32. doi: 10.1042/bj1180229, PMID 5484666.

17. Bancroft JD, Gamble M. Theory and practice of histological techniques. Elsevier health sciences; 2008.

18. Gibson JD, Pumford NR, Samokyszyn VM, Hinson JA. Mechanism of acetaminophen-Induced Hepatotoxicity: Covalent Binding versus Oxidative Stress. Chem ResToxicol. 1996;9(3):580-5. doi: 10.1021/tx950153d, PMID 8728501.

19. Thabrew MI, Joice PDTM, Rajatissa WA. A comparative study of the efficacy of Pavetta indica and Osbeckia octandra in the treatment of liver dysfunction. Planta Med. 1987;53(3):239-41. doi: 10.1055/s-2006-962691, PMID 3628555

20. Ayala A, Muñoz MF, Argüelles S. Lipid peroxidation: Production, metabolism, and signaling mechanisms of malondialdehyde and 4-hydroxy-2-nonenal. Oxid Med Cell Longev. 2014;2014:360438. doi: 10.1155/2014/360438.

21. Gaschler MM, Stockwell BR. Lipid peroxidation in cell death. Biochem Biophys Res Commun. 2017;482(3):419-25. doi: 10.1016/j.bbrc.2016.10.086, PMID 28212725.

22. Cioffi F, Adam RHI, Broersen K. Molecular mechanisms and genetics of oxidative stress in Alzheimer's disease. J Alzheimers Dis. 2019:72(4):981-1017. doi: 10.3233/JAD-190863, PMID 31744008.

23. Hendrix J, Nijs J, Ickmans K, Godderis L, Ghosh M, Polli A. The interplay between oxidative stress, exercise, and pain in health and disease: Potential role of autonomic regulation and epigenetic mechanisms. Antioxidants (Basel). 
2020;9(11):1166. doi: 10.3390/antiox9111166, PMID 33238564

24. Li S, Hong M, Tan HY, Wang N, Feng Y. Insights into the role and interdependence of oxidative stress and inflammation in liver diseases. Oxid Med Cell Longev. 2016;2016:4234061. doi: 10.1155/2016/4234061.

25. Drotman RB, Lawhorn GT. Serum enzymes as indicators of chemically induced liver damage. Drug Chem Toxicol. 1978;1(2):163-71. doi:
10.3109/01480547809034433, PMID 755666.

26. Ramachandran A, Jaeschke H. Acetaminophen hepatotoxicity. Semin Liver Dis. 2019;39(2):221-34. doi: 10.1055/s-0039-1679919, PMID 30849782.

27. McCrae JC, Morrison EE, MacIntyre IM, Dear JW, Webb DJ. Long-term adverse effects of paracetamol - a review. Br J Clin Pharmacol. 2018;84(10):2218-30. doi: 10.1111/bcp.13656, PMID 29863746

Article History: Received: 15-10-2021; Revised: 23-11-2021; Accepted: 03-12--2021

Cite this article: Kataki MS, Kakoti BB. An Optimized Herbal Formula Reverses the Hepatotoxicity Induced by Acetaminophen. J Young Pharm.

$2022 ; 14(1): 56-61$. 